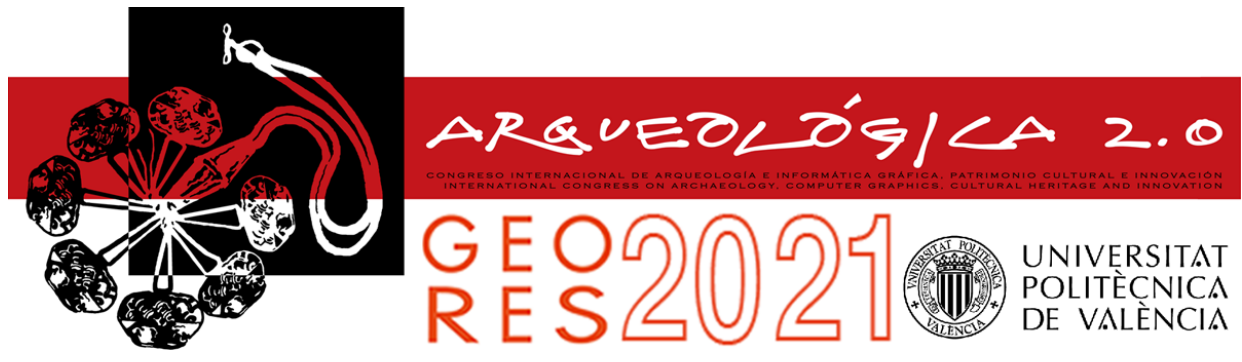

Proceedings of the joint international event $9^{\text {th }}$ ARQUEOLÓGICA

$2.0 \& 3^{\text {rd }}$ GEORES

Valencia (Spain).

26-28 April 2021

\title{
DIGITAL TWINS: COMBINED SURVEYING PRAXIS FOR MODELLING
}

\author{
Cecilia Maria Bolognesi", Martina Signorini \\ Department of Architecture, Built Environment and Construction Engineering, Politecnico di Milano, Via G. Ponzio 31, 20133 Milan, Italy. \\ cecilia.bolognesi@polimi.it; martina.signorini@polimi.it
}

\begin{abstract}
:
While the construction sector embraces the digitalization, new technologies are spreading and are generating benefits. The need of creating a 3D model of the reality, in particular of the built asset, is not new. The Building Information Modelling, a process that gives a great contribution in improving project quality, reducing errors, avoiding uncertainties and enhancing collaboration, allows a virtual representation of the existing asset enriching its geometry with precious and significant information related to its properties. Despite BIM benefits, BIM models do not take into account the real-time component and do not report the real-time behaviour of the building. Digital twin, the virtual copy of an object, instead creates a realtime virtual twin of the physical asset considering this ingredient and reproducing how the building behaves. The paper starts right from the investigation of the Digital Twin concepts and its main features and proceeds with an analysis of several technologies and instruments exploited till now for the surveying and positioning of existing buildings. In addition, a new toolkit based on AR and coupled with sensors and visualisation tool developed by BIM4EEB, an ongoing H2020 project, is presented to show its main advantages when it comes to representing the virtual copy of an existing building.
\end{abstract}

Keywords: digital twin, virtual reality, BIM, digitalization, survey

\section{Introduction}

Nowadays the AEC (Architecture, Engineering and Construction) sector is fully involved in the digitalization focusing on the management of data and the interconnection between machines-objects-people and processes. Information in real environments, data management (big data and $\mathrm{Al}$ ), digital collaboration and intelligent objects (IoT) are the keywords of this revolution (Daniotti et al., 2020).

With BIM, a widely used concept in the literature and implemented in real-world construction projects around the globe, the construction sector has had the chance to improve productivity and quality in building but also in infrastructure projects, reducing both design and construction errors and financial losses, avoiding uncertainties thanks to the interoperability among all stakeholders and ICT tools. Despite BIM potentialities and although BIM uses cover lifecycle management of built asset, BIM models don't consider how people interact with the built environment and they don't reproduce real-time behaviour of the asset in service or in use (Matarneh, Danso-Amoako, Al-Bizri, Gaterell, \& Matarneh 2019). Sensors and IoT devices would perform the function of enhancing the information set of high-fidelity BIM models by providing real-time data streaming with a consequent improvement in construction and operational effectiveness. However, BIM and loT integration are still in progress (Tang, Shelden, Eastman, Pishdad-Bozorgi, \& Gao, 2019).

Digital Twin - whose first adoption dates back to 2010 when NASA used it in technology roadmaps and proposals for exploration of sustainable space (Caruso, Dumbacher, \& Grieves, 2010; National Research Council, 2012) and then in fighter aircrafts and NASA vehicles (Glaessgen \& Stargel, 2012; Tuegel, Ingraffea, Eason, \& Spottswood, 2011) - tries to achieve what BIM models have not delivered. Based on the idea that a digital informational construct about a physical asset could be created as an entity on its own and linked with that physical system through its whole life cycle, the digital representation should include all information (Burnett et al., 2019) concerning the system asset that could be potentially obtained from its thorough inspection in the real world (Grieves \& Vickers, 2016).

Although there is the lack of a universal definition about what a Digital Twin is for application to a construction or infrastructure asset, it is possible to outline its main features and potentialities.

From the definition of Grieves according to which the Digital Twin is defined as "a set of virtual information constructs that fully describes a potential or actual physical manufactured product from the micro atomic level to the macro geometrical level" (Grieves \& Vickers, 2016) to another significant definition highlighting the importance of Digital Twin over BIM by Stojanovic

"Corresponding Author: Cecilia Maria Bolognesi, cecilia.bolognesi@polimi.it 
(Stojanovic, Trapp, Richter, Hagedorn, \& Döllner, 2018) stating that "a Digital Twin (DT) is a digital duplicate of the physical environment, states and processes. While a BIM model contains as-is and historical data, a DT can be used to assess the current state, and to potentially forecast the future state", it is clear what digital twin represents.

Following the approach of Grieves, the Digital Twin concept model is composed by three main parts:

a) physical product;

b) virtual product;

c) data and information that provides the connections between the virtual and the real.

The connection among the virtual product and the physical product is provided by data in different forms. The Physical collects and store real time data that are sent to virtual copy for processing. Vice versa, the Virtual applies its imbedded engineering models and $\mathrm{Al}$ subjecting data to transformations and processing information (Fig. 1) (Grieves, 2015).

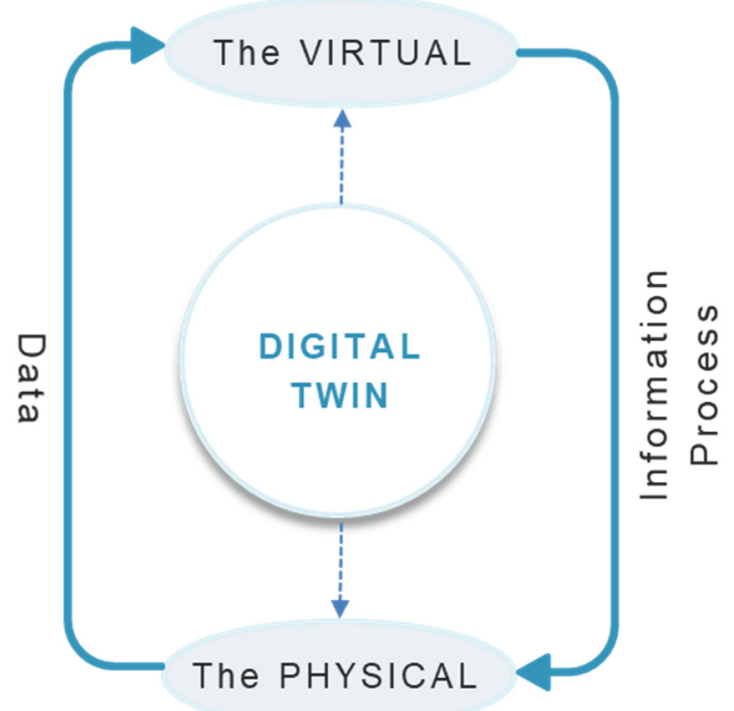

Figure 1: The Digital twin concept.

It is fundamental to underline the strong interconnection among the Physical and the Virtual: in fact, the digital twin is not only the copy of the real asset, but it is also strictly connected to it by specific relationships.

The supporting technologies for the DT development are: wireless sensor networks, installed in buildings to collect data about temperature, humidity, lighting, occupancy, etc. and data analytics, a broad term including the artificial intelligence A.I. and machine learning concepts (Khajavi, Motlagh, Jaribion, Werner, \& Holmstrom, 2019).

The virtual copy can be visualized in a $3 \mathrm{D}$ model, but thanks to the use of various sensor networks a real-time view of the built asset is created (Fig. 2). The dynamic view allows for real-time analysis, building efficiency and comfort enhancement and informed decision-making (Khajavi, Motlagh, Jaribion, Werner, \& Holmstrom, 2019).

Concerning the level of development, the twin could vary from a simple component, moving to a building or even country such as the one implemented by the UK National
Digital Twin programme (Centre for Digital Built Britain, 2020).

Proceeding towards the digitalization of the construction sector, embracing the digital twin technology and applying it to the existing construction means a series of benefits such as construction and operating costs reduction, productivity and collaboration enhancement, safety improvement, asset performance and sustainability optimization.

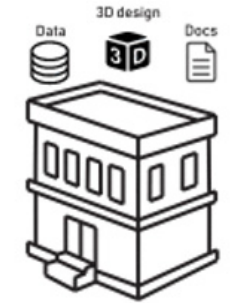

$$
\text { Required Data Components from }
$$<smiles>[CH]</smiles>
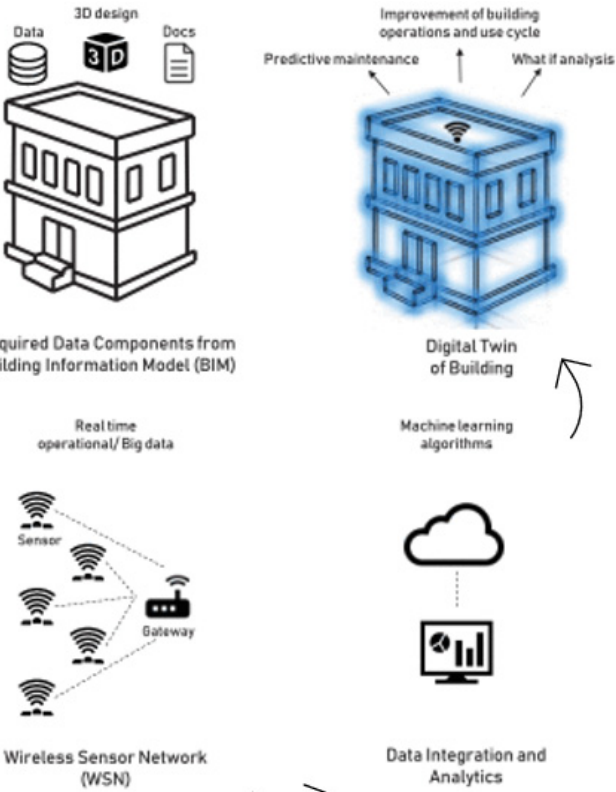

Data Integration and Analytics
Figure 2: Components to create a digital twin of building and comparison with BIM (Khajavi, Motlagh, Jaribion, Werner, \& Holmstrom, 2019).

This paper investigates how a virtual copy, named a digital twin, of an existing building can be reproduced, analysing which are the main used tools among the existing ones and outlining the features of the AR-toolkit developed by the $\mathrm{H} 2020$ ongoing project BIM4EEB.

The paper is organized as follows. After an introduction dealing with the concept of digital twin, section 2 focuses on the common instruments for the surveying and positiong of the model, section 3 introduces a new tool based on Augemented Reality coupled with sensors developed by the BIM4EEB project. Section 4 summarises the outcomes and the future developments.

\section{Surveying and positioning}

The construction of a digital model of an existing or historical building (Dore, \& Murphy, 2017) brings with it the need for a phase of accurate survey, that is to say of real importance; the technological evolution that has followed over the last twenty years has led in the first instance to the development of various hardware, software and devices for the measurement and acquisition of the existing building and immediately afterwards to the translation of the information into what is defined a BIM model (Osello, Lucibello, \& Morgagni, 2018). The measurement themes focus on the conversion of the different units according to the instrument used, the translation of the information into the model on the difficulty of spatial positioning of the information. 


\subsection{Surveying}

Among the existing tools for surveying, technologies are multiplying, specialising in relationship with the size of surveying objects and their visibility inside the building also. What can be precisely detected on a surface by one tool can be ignored by another; on the contrary, what is inserted inside a masonry is ignored for example by a laser or photogrammetric techniques. Depending on the peculiarities of each one a survey of the building could have to do with:

- $\quad$ Lidar technologies (Light Detection and Ranging or Laser Imaging Detection and Ranging) that allow to reconstruct three-dimensional models through the recording of single or multiple scans determining the distance of an object or surface using a laser pulse, based on the time of flight (the time it takes for the laser beam to travel to the target and reflect back) (Cardaci, Mirabella Roberti, \& Versaci, 2019);

- Photogrammetry, a passive detection system, time consuming in terms of post processing but with undoubted economic advantages and increasingly faster considering the development of the latest modeling software algorithms (Remondino, Nocerino, Toschi, \& Menna, 2017);

- Infrared thermometers as well as heat cameras where thermal mappings give different information about the building such as objects behind others with uniform temperature, find heat leaks, or detect faulty electric cabling and which can rarely be associated with model geometries except with the intervention of the operator (González-Jorge, Lagüela, MartínezSánchez, 2012);

- Radar which uses electromagnetic radiation in the microwave band (UHF/VHF frequencies) of the radio spectrum, and detects the reflected signals from subsurface structures;

- Magnetic or x-ray sensors but also capacitors or voltage or stud detectors as well as $\mathrm{x}$-rays or

- ultrasounds where ultrasonic range sensors produce a beam of ultrasound that is sent out and reflects back from the object, allowing the sensor to measure distances but different density of a wall as well. This technology is the same used in medicine application to create multidimensional images of the human body, detecting different densities.

Considering the different structure and nature of data acquisition it is natural to read in digital twin the only possibility to collect them, assuming the model as the unique container and the digital replica of what we have investigated. Therefore, in addition to the difficulties derived from the different previous measurements, there are also those derived from the need to position the measurement we are performing.

\subsection{Positioning}

After surveying the main task to organize the digital replica could be concerning both the spatial positioning of the model within a Coordinate System and an Internal Positioning System of the surveys carried out. In the case of heat cameras for example this association can be done manually. In the case of some laser scanners the connected applications allow the automatic recording of the user's movements from one scan to another for prerecording in the field without manual intervention but also the acquisition of associated images in HDR (High Dynamic Range) (Pavan et al., 2020). However, in the case of more precise detection positioning, it may be necessary to scale down the indoor positioning down to 1:50 detail, with tolerances of just a few $\mathrm{mm}$.

Almost all indoor positioning systems also lack external reference systems due to their nature and are anchored to temporary positioning systems that should be linked to more general systems. In fact, while for the outdoor environment, the GPS geolocation system is particularly effective, inside buildings or in heavily urbanized areas, the GPS easily loses operation, and it is necessary to play hard to find other alternatives.

Among the most used technologies for indoor positioning, we consider with different funcionalities:

- Beacon as other technologies or app for mobile devices have found wide use both in maintenance and in areas more related to Cultural Heritage as tracking tools. Specifically beacons are hardware devices thanks to which bluetooth technology is used to send and receive signals within short distances. They are used nearby as access-points to calculate where the device is located.

- Glasses: Hololens, HTC Vive, Oculus Rift. They are different in functionality with respect to which they have been designed but all three disposals contain positioning capabilities with respect to indoor environments. Hololens, designed for AR for the vision of holograms superimposed on environments, includes several sensors, to measure inertia also, a light sensor and four cameras for environmental analysis. The tool measures the time of flight for IR light and creates a $3 d$ image of the room. The accuracy is about $3 \mathrm{~cm}$. HTC vive uses an IR-flash, little microchips with a photocell that are tuned to listen to infrared light, followed by sweeping IRline lasers horizontal and vertical. It is a passive sensor sensing on headset, handles and trackers. Positioning is then determined by detecting the pulses of a photo led. The time between the initial pulse and the pulse generated by the line laser helps the positioning with a $2 \mathrm{~mm}$ accuracy with a $30 \mathrm{~Hz}$ update time. There are some limitations in the dimension of the spaces that must be related to the sensor's possibility of detection. Set up considers both PC and Headset. Oculus rift uses LED markers on the headset, handles and trackers and then uses 
cameras to track their position. The cameras are both fitted in the headset as well as the on stands in the room. The accuracy of this system is around 3.5 to $12 \mathrm{~mm}$ but just as with the HTC vive errors increase on distance to the cameras.

- QR codes attached to objects is the most traditional tool we can use but still one of the most accurate. A simple camera tracks objects with a camera. If the $Q R$ code is attached to a fixed object as a reference point geographically that position will be known for a camera. The orientation, angle and the position of the camera can be calculated by register the symmetry and size of the QR-code in the image. Image recognition is used in AR-tools and this allows to anchor/position a virtual CAD to the real world so that they match each other.

\section{The BIM4EEBproject}

BIM4EEM is a wide $\mathrm{H} 2020$ project coordinated by Politecnico of Milan with the participation of fifteen partners spread all over Europe. The aim of the project is to foster the renovation industry by developing an attractive and powerful BIM-based toolset able to support designers in the design and planning phase, construction companies to efficiently carry out the work and service companies to provide attractive solutions for building retrofitting. A principal tool eases decision making and asset management, thanks, among all, to the exploitation of augmented reality.

Through the BIM management system linked data and a set of tools increases semantic interoperability between software and stakeholders involved along the overall renovation process (design, planning, construction, performance assessment and management).

The renovation project within the project is carried out according to the expectations and requests of the renovation market; the project considers two public administrations, and two general contractor companies will validate the toolset in a social housing setting and private residential buildings in Italy, Poland and Finland.

In this field a tool has been developed to facilitate the creation of digital twins starting from the existing construction and for which the detection operations would meet parameters of accuracy, speed, transfer within an interoperable data management system.

\subsection{The fast mapping toolkit}

The aim of the digital renovation process deepens how to get knowledge about the building and create a useful model for a more efficient and safe renovation process.

With the experimentation the partners wish to make up for the shortcomings of many devices where a wide abundance of sensor technologies available lacks interfaces and interoperability for collecting the data measured or lack shareable positioning systems.

The BIM4EEB project includes developing of tools to implement the mapping of buildings; the aim is to reach a faster workflow that includes the detection of hidden installations and materials in the wall that will help the renovation process both in recent than more ancient building. The approach followed has been to be able to use devices and solutions starting from tools and software already available on the market but which, in new couplings, can meet the needs of speed, accuracy and survey of hidden parts (Fig. 3).

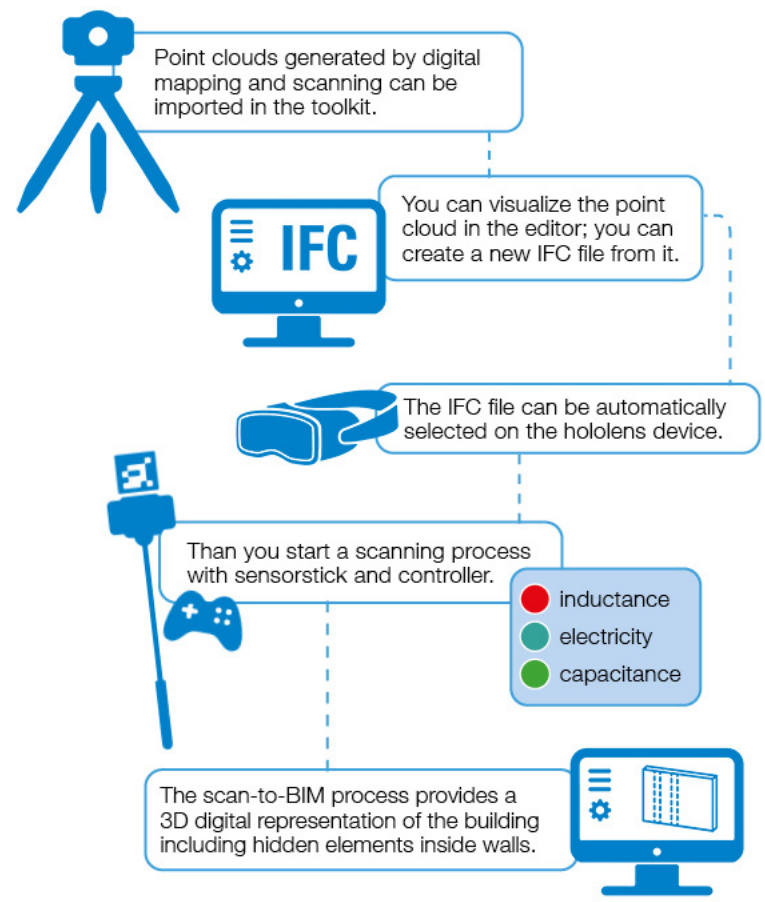

Figure 3: The figure shows the workflow from scanning and mapping to visualize objects behind hidden surfaces in buildings.

From one side mapping tools as previously seen, from the other 3D-laser scanning that measure the geometry, are the beginning of the workflow that deals with collected data to be stored and translated by a BIM-platform into a 3D-model and visualized for the user.

The aims to process and transform the whole data into a BIM model, saving time, mapping process with quality, and storing data easily, works on IFC-format as a requirement of the whole process.

The experimentation uses a combination of market available laser scanning techniques with a HoloLens device and a prototype sensor array consisting of heat sensor, stud detectors and more as mapping equipment. The so called -sensor stick- measure capacitance, temperature, inductance, voltage and position so that it's possible to visualize the room with its hidden installations.

Preparation and equipment for setup includes the positioning system and markers when needed.

The sensor dialogues through a sensor stick with several functions for detecting installations inside walls with Hololens combining input data directly into the point cloud previously surveyed. But both mapping data are converted in a 3D model in Unity environment to dialogue and be transformed into an IFC-file.Through the HoloLens the user can see the physical environment around as well as a digital representation of the scanned data. At the beginning of the process a calibration procedure needs to be done for the HoloLens view to align with the 3D laser 
scan and the sensor stick (Fig. 4). During the process different spaces can be added one after the other while the building data is mapped in a hierarchy, defining different parts such as floors, walls.

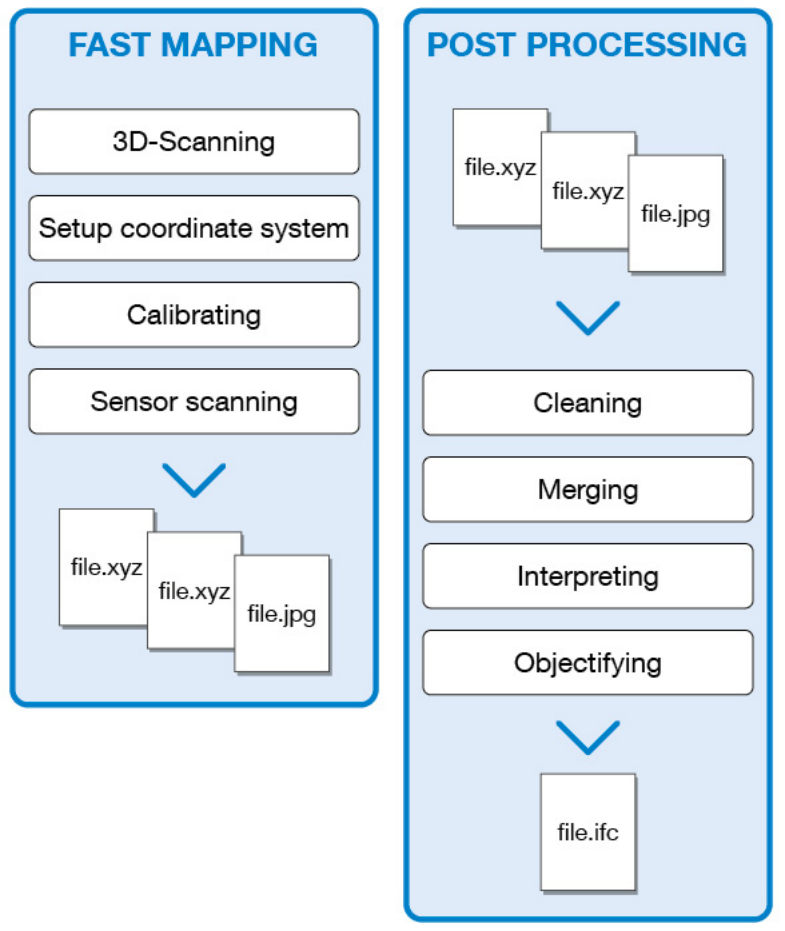

Figure 4: Survey with the new set of fast mapping and post processing operation.

The view of the point cloud in the headset obliges to optimize the point cloud whose import would result impossible in the Hololens device. For this reason, the point cloud is streamed and not imported, even if this approach is under investigation now. One service has been organized for streaming point cloud data, the other for altering IFC-files and to provide a visualization model for the AR-device. A dedicated app has been developed for managing and converting point clouds and provide a visualization.

\section{Future developments}

As briefly outlined in this paper, the presence of increasingly sophisticated hardware is fundamental for the collection of data useful for the creation of a digital twin. The experience of the Fast Mapping Toolkit developed in BIM4EEB project is an example of this and indicates several possibilities of deepening and optimizing workflows. There are some topics that will become the object of future developments that will have to answer typical questions related the surveying process in order to elaborate a clear digital replica such as: the size of the data bases of the surveys, the translation into interoperable languages, the possibility of integration between different surveying units, the portability of the tools, the cost-effectiveness of the system.

These and other topics will be developed in the next steps of the projects.

\section{Acknowledgements}

This paper deals with one specific part of the BIM4EEB project, $x x x$, responding to the Call for proposals LC-EEB02-2018 Building information modelling adapted to efficient renovation. The project has received funding from European Union's H2020 research and innovation programme under grant agreement $\mathrm{N}$. GA $\mathrm{N}$. $82066030 / 06 / 2020$. The content of this document reflects only the author's view only and the Commission is not responsible for any use that may be made of the information it contains.

The development of the Sensor stick has been led mainly by RISE and CGI Sweden partner; and in the final project deliverable, confidential still, by Deliverable 5.3.

\section{References}

Burnett, D., Thorp, J., Richards, D., Gorkovenko, K., \& Murray-Rust, D. (2019). Digital twins as a resource for design research. Proceedings - Pervasive Displays 2019 - 8th ACM International Symposium on Pervasive Displays, PerDis 2019, 6-7. https://doi.org/10.1145/3321335.3329685

Caruso, P. W., Dumbacher, D. L., \& Grieves, M. W. (2010). Product Lifecycle Management and the quest for sustainable space exploration. AIAA SPACE Conference and Exposition 2010, 1-12. https://doi.org/10.2514/6.2010-8628

Cardaci, A., Mirabella Roberti, G., \& Versaci, A. (2019).The integrated 3D survy for planned conservation: the former church and convent of sant'Agostino in Bergamo. Int. Arch. Photogramm. Remote Sens. Spatial Inf. Sci., XLII-2/W9. (pp. 235-242). https://doi.org/10.5194/isprs-archives-XLII-2-W9-235-2019

Centre for Digital Built Britain. (2020). National Digital Twin programme (NDTp). Retrieved March 15, 2021, from https://www.cdbb.cam.ac.uk/what-we-do/national-digital-twin-programme

Daniotti, B., Pavan, A., Lupica Spagnolo, S., Caffi, V., Pasini, D., \& Mirarchi, C. (2020). Collaborative Working in a BIM Environment (BIM Platform). In BIM-Based Collaborative Building Process Management (pp. 71-102). Springer International Publishing. https://doi.org/10.1007/978-3-030-32889-4

Glaessgen, E. H., \& Stargel, D. S. (2012). The digital twin paradigm for future NASA and U.S. Air force vehicles. Collection of Technical Papers - AIAA/ASME/ASCE/AHS/ASC Structures, Structural Dynamics and Materials Conference, April. https://doi.org/10.2514/6.2012-1818

González-Jorge, H., Lagüela, S., Martínez-Sánchez, J. (2012).Single image rectification of thermal images for geometric studies in façade inspections. Infrared Physics and Technology. https://doi.org/ 10.1016/j.infrared.2012.05.003

Grieves, M. (2015). Digital Twin: Manufacturing Excellence through Virtual Factory Replication. White Paper, March. available 
https://www.researchgate.net/publication/275211047_Digital_Twin_Manufacturing_Excellence_through_Virtual_Fa ctory_Replication

Grieves, M., \& Vickers, J. (2016). Digital Twin: Mitigating Unpredictable, Undesirable Emergent Behavior in Complex Systems. In F.-J. Kahlen, S. Flumerfelt, \& A. Alves (Eds.), Transdisciplinary Perspectives on Complex Systems (pp. 85-113). Springer. https://doi.org/https://doi.org/10.1007/978-3-319-38756-7_4

Khajavi, S. H., Motlagh, N. H., Jaribion, A., Werner, L. C., \& Holmstrom, J. (2019). Digital Twin: Vision, benefits, boundaries, and creation for buildings. IEEE Access, 7, 147406-147419. https://doi.org/10.1109/ACCESS.2019.2946515

Matarneh, S. T., Danso-Amoako, M., Al-Bizri, S., Gaterell, M., \& Matarneh, R. (2019). Building information modeling for facilities management: A literature review and future research directions. Journal of Building Engineering, 24(January), 100755. https://doi.org/10.1016/j.jobe.2019.100755

Dore, C., \& Murphy, M.(2017). Current state of the art historic building information modelling. Int. Arch. Photogramm. Remote Sens. Spatial Inf. Sci., XLII-2/W5. (pp.185-192), https://doi.org/10.5194/isprs-archives-XLII-2-W5-1852017.

National Research Council. (2012). Technology Area 12: Materials, Structures, Mechanical Systems, and Manufacturing Road Map. Available at: https://www.nap.edu/read/13354/chapter/22\#296

Osello, A., Lucibello, G., \& Morgagni, F.(2018). HBIM and Virtual Tools: A New Chance to Preserve Architectural Heritage. Buildings (pp. 8-12). https://doi.org/10.3390/buildings8010012

Pavan, A., Bolognesi, C., Guzzetti, F., Sattanino, E., Pozzoli, E., D’Abrosio, L., Mirarchi, C., \& Mancini, M (2020) BIM Digital Platform for First Aid: Firefighters, Police, Red Cross. In: Daniotti B., Gianinetto M., Della Torre S. (eds) Digital Transformation of the Design, Construction and Management Processes of the Built Environment. Research for Development. Springer, Cham. https://doi.org/10.1007/978-3-030-33570-0_25

Remondino, F., Nocerino, E., Toschi, I., \& Menna, F.(2017). Acritical review of automated photogrammetric processing of large dataset. Int. Arch. Photogramm. Remote Sens. Spatial Inf. Sci., XLII-2/W5. (pp. 591-599) https://doi.org/10.5194/isprs-archives-XLII-2-W5-591-2017

Stojanovic, V., Trapp, M., Richter, R., Hagedorn, B., \& Döllner, J. (2018). Towards the generation of digital twins for facility management based on 3D point clouds. Proceeding of the $34^{\text {th }}$ Annual ARCOM Conference, ARCOM 2018, June, 270-279.

Tang, S., Shelden, D. R., Eastman, C. M., Pishdad-Bozorgi, P., \& Gao, X. (2019). A review of building information modeling (BIM) and the internet of things (IoT) devices integration: Present status and future trends. Automation in Construction, 101(January), 127-139. https://doi.org/10.1016/j.autcon.2019.01.020

Tuegel, E. J., Ingraffea, A. R., Eason, T. G., \& Spottswood, S. M. (2011). Reengineering aircraft structural life prediction using a digital twin. International Journal of Aerospace Engineering. https://doi.org/10.1155/2011/154798 